\title{
Transjugular Intrahepatic Portosystemic Shunt Using the Trans-splenic Approach and a Snare Technique
}

\author{
Juil Park ${ }^{1}$ Hyo-Cheol Kim ${ }^{1}$ \\ ${ }^{1}$ Department of Radiology, Seoul National University Hospital, \\ Seoul, Korea \\ J Clin Interv Radiol ISVIR 2017;1:175-178.
}

\author{
Address for correspondence Hyo-Cheol Kim, MD, Department of \\ Radiology, Seoul National University Hospital, 101 Daehak-ro, \\ Jongno-gu, Seoul, 110-744, Korea \\ (e-mail: angiointervention@gmail.com).
}

\author{
Abstract \\ Keywords \\ - transjugular \\ intrahepatic \\ portosystemic shunt \\ - trans-splenic \\ approach \\ - portal vein \\ thrombosis
}

Transjugular intrahepatic portosystemic shunt (TIPS) is an established procedure for the secondary prevention of variceal bleeding and management of refractory ascites. Portal vein thrombosis presents technical difficulty during TIPS due to nonvisualization of portal vein. In such patients, trans-splenic approach can be utilized to navigate thrombosed portal vein and use a snare as a fluoroscopic guidance for the intraparenchymal pass. We present the case of a 63-year old man who received the successful TIPS procedure via trans-splenic access without any complication.

\section{Introduction}

The main indication of transjugular intrahepatic portosystemic shunt (TIPS) is the treatment of variceal bleeding and refractory ascites in cirrhotic patients. In the presence of portal vein thrombosis in patients with cirrhosis, the risk of variceal bleeding increases and portal vein access during the procedure can be difficult. Various approaches including transhepatic, trans-splenic approach, and transmesenteric ileocolic vein have been utilized to create TIPS in patients with portal vein thrombosis.

We have recently had a patient who suffered from intractable ascites and right portal vein thrombosis. Transjugular approach was tried to create TIPS, but it failed. Subsequently, TIPS was created by trans-splenic approach with a snare technique.

\section{Case Report}

A 63-year-old male patient presented with intractable ascites. Ultrasonography (USG) and computed tomographic (CT) scan demonstrated features of cirrhosis and massive ascites. Bland thrombus was noted in the right portal vein with the peripheral segmental portal vein in right lobe

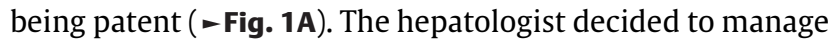
massive intractable ascites by creation of TIPS in conjunction with the interventional radiology team.

A 9F sheath (Cook, Bloomington, Indiana, United States) was inserted via right internal jugular vein. After selection of the right hepatic vein with a multipurpose angled catheter, the TIPS needle (Colapinto needle; Cook) was advanced toward the right portal vein, which failed to puncture the portal vein. The right common femoral artery was accessed, and angiography was performed via the celiac trunk and superior mesenteric artery. Delayed images of superior mesenteric angiogram were not able to demonstrate clearly the right portal vein due to thrombosis. Next, microcatheter (Microferret; Cook) was advanced into the right posterior hepatic artery, and the TIPS needle was advanced toward the right portal vein by using the microcatheter as a target, which failed as well.

Next day, ultrasound-assisted percutaneous trans-splenic puncture of the perihilar splenic vein was performed by using a 2G Chiba needle (Cook). After access was achieved, a received

April 13, 2017

accepted after revision

May 1, 2017

published online

September 5, 2017
DOI https://doi.org/

10.1055/s-0037-1603830. ISSN 2457-0214.
Copyright (e) 2017 by Indian Society of

Vascular and Interventional Radiology
License terms

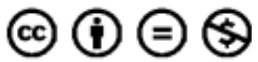


A
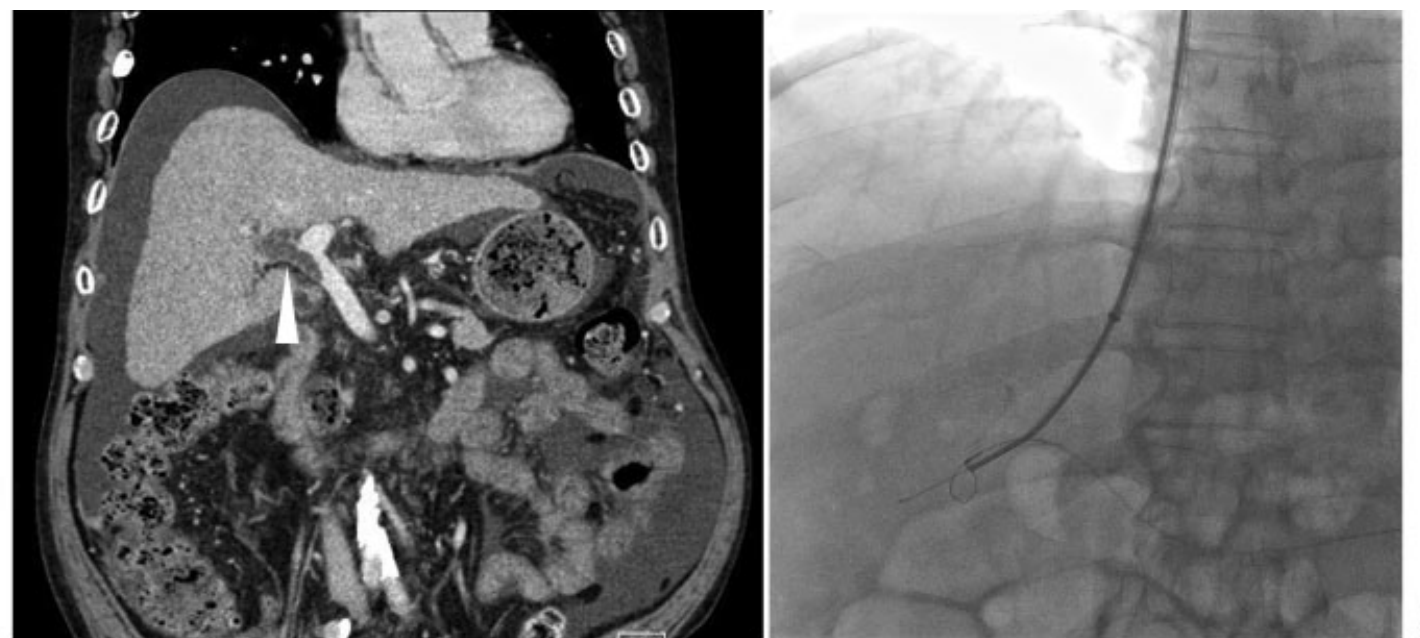

C

B
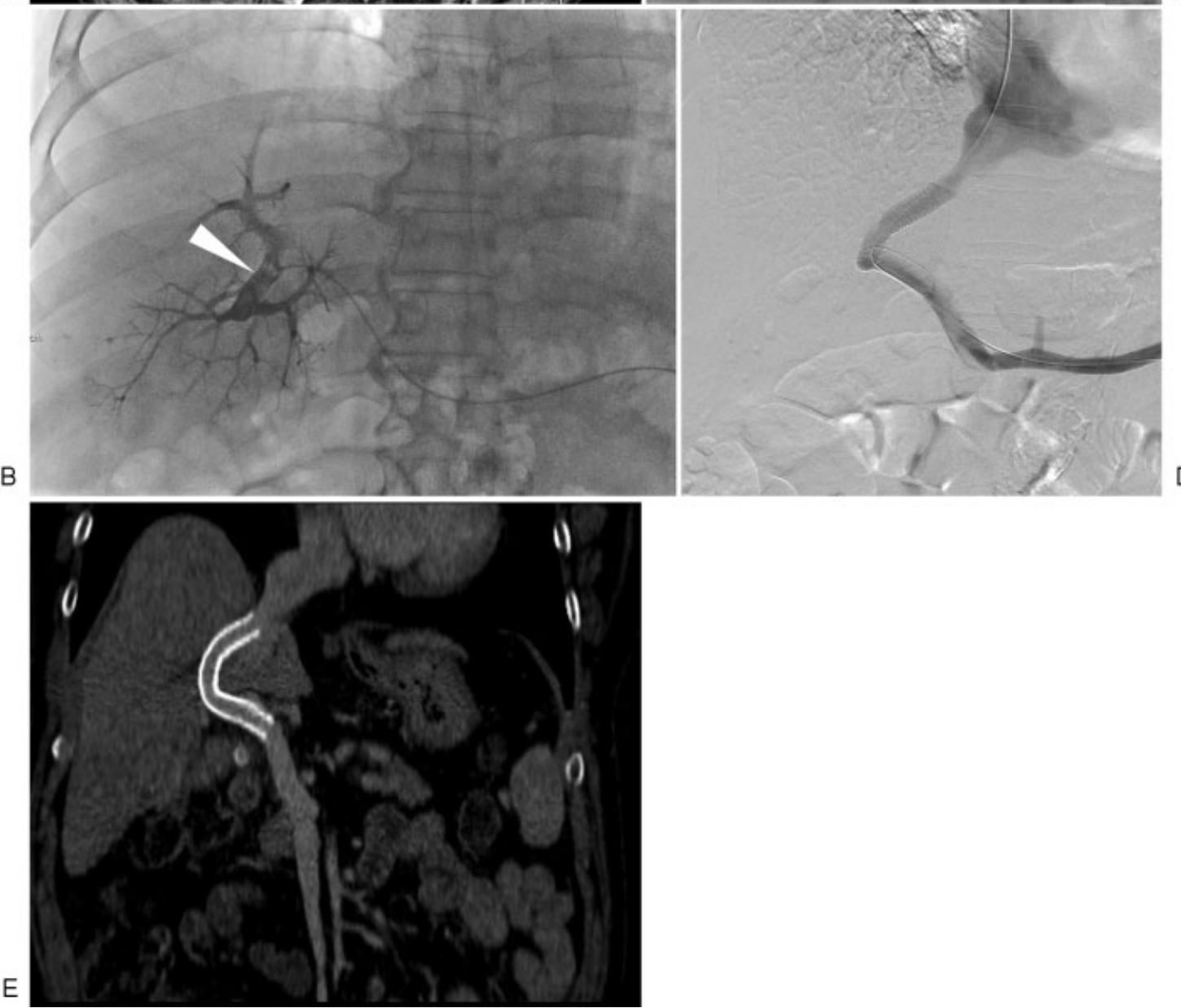

Fig. 1 A 63-year-old male patient presented with intractable ascites. (A) Coronal enhanced CT scan shows thrombosis (arrowhead) in right portal vein and main portal vein. Abundant ascites is noted. (B) $5 \mathrm{~F}$ multipurpose catheter was advanced into the right portal vein via transsplenic access. Right posterior portal vein (arrowhead) was filled with contrast media. (C) After placement of a snare in the right posterior portal vein, Colapinto needle was advanced through the snare and guidewire was inserted into the peripheral portal vein. (D) Splenic venography shows good flow through the TIPS. (E) Coronal reformatted enhanced CT scan 1 year after TIPS creation shows patent TIPS.

0.018-in wire was advanced into the splenic vein. The $5 \mathrm{~F}$ Accu-introducer catheter (Sungwon Medical, Seoul, South Korea) was advanced over the 0.018 -in wire. A 0.035 -in hydrophilic guidewire (Terumo, Tokyo, Japan) was advanced through the Accu-introducer catheter. A 6F sheath was placed over a 0.035 -in wire.
Splenic venography was performed to visualize the portal vein, and a $5 \mathrm{~F}$ catheter was advanced into the right portal vein (-Fig. 1B). Peripheral segmental portal vein in the right lobe was patent and right portal vein was thrombosed. A 10-mm snare (EV3 Endovascular, Plymouth, Minnesota, United States) was advanced in the peripheral third-order 
portal vein. The snare was used as the target for the TIPS needle. The TIPS needle penetrated through the snare (-Fig. 1C), an exchange length wire was advanced via the trans-splenic route into the peripheral portal vein, which was then was grabbed by a snare. Through-and-through wire was established from the internal jugular vein to splenic access ( - Video 1 ). Over the wire, a $4 \mathrm{~F}$ catheter was advanced and indwelling guidewire exchanged for a stiff wire (Amplatz; Cook). The intrahepatic puncture tract was dilated by $8-\mathrm{mm}$ $\times 4$-cm balloon (Mustang; Boston Scientific, Natick, Massachusetts, United States), and a $10-\mathrm{mm} \times 10$-cm Viabahn stentgraft (Gore, Flagstaff, Arizona, United States) was placed (-Fig. 1D). Pressure gradients between the main portal vein and inferior vena cava were 27 and $8 \mathrm{~mm} \mathrm{Hg}$ before and after TIPS creation, respectively.

\section{Video 1}

Guidewire was captured by a snare, and through-andthrough wire was established. Online content viewable at: https://www.thieme-connect.com/ products/ejournals/html/doi/10-1055-s-00371603830-jcir-17-00037-v1.mp4.

Hemostasis of trans-splenic access was achieved by using $8 \mathrm{~mm} \times 14-\mathrm{cm}$ coil (Nester; Cook) and a mixture of n-butyl cyanoacrylate (Histoacryl; B. Braun, Melsungen, Germany) and iodized oil (Lipiodol; Guerbet, Aulnay-sous-Bois, France) with a 1:2 mixing ratio. Massive ascites was well controlled after TIPS creation, and TIPS was patent on enhanced CT scan 1 year later (-Fig. 1E).

\section{Discussion}

TIPS procedure has been used to treat the many complications associated with the portal hypertension. Secondary prevention of variceal bleeding and refractory ascites are the two main indications for which the effectiveness of TIPS has been studied with the randomized controlled trials. ${ }^{1}$ The meta-analysis published in 2008 reported significant reduction in variceal rebleeding and death due to rebleeding while all-cause mortality rates were similar to that of endoscopic therapy for variceal bleeding. ${ }^{2}$ TIPS was superior to the largevolume paracentesis with albumin supplementation in the control of the refractory ascites and had better survival rates. ${ }^{3}$ However, because of the direct shunt created between the portal and the systemic circulation that bypasses the hepatic parenchyma, hepatic encephalopathy develops or worsens after TIPS. ${ }^{1}$

Intrahepatic access to the portal venous system is required in the creation of TIPS and is usually acquired via percutaneous transjugular intrahepatic access. ${ }^{4}$ In cases of nonvisualized intrahepatic portal vein, or main portal vein occlusion, the percutaneous trans-splenic access may provide the access to the portal vein. Trans-splenic approach was introduced in 1951, but it was soon abandoned due to the risk of bleeding from the splenic puncture site. However, after Probst et $\mathrm{al}^{5}$ reported the prevention of bleeding by plugging the splenic tract with a compressed absorbable gelatin sponge, splenoportography is considered a safe procedure. ${ }^{4}$ Although the endovascular treatment via percutaneous trans-splenic access is still controversial, the recent studies reported the safe and successful endovascular treatment of vascular complications in patients with chronic liver disease. ${ }^{4,6}$

In cases of portal vein thrombosis, hepatic venography from the transjugular approach does not readily reveal the portal vein, thus making the intrahepatic access difficult. However, with the trans-splenic approach, thrombosed portal vein can be carefully navigated using faint opacification of the fibrotic cord of the thrombosed portal vein as the fluoroscopic guidance. Then TIPS needle is advanced toward the snare located in the thrombosed portal vein as a fluoroscopic target in careful manner using oblique views rather than the parenchymal pass in a single motion as used in the conventional TIPS procedure. Then an exchange length stiff wire is advanced through the TIPS needle and withdrawn through the splenic sheath, thus making through-andthrough access in which TIPS procedure can be performed. ${ }^{7}$

The previous study on the efficacy of portal vein recanalization and TIPS placement (PVR-TIPS) in patients with chronic obliterative portal vein thrombosis reported $100 \%$ technical success rate in all 20 patients in whom the transsplenic approach was utilized. ${ }^{7}$ Among 60 patients who received PVR-TIPS via either transhepatic or trans-splenic approaches, recanalized portal vein and TIPS maintained patency in 55 patients (92\%) during the follow-up or to the time of transplant. ${ }^{7}$ Another prospective study with 11 patients with main portal vein thrombosis who were denied of the liver transplant due to portal vein thrombosis reported $100 \%$ technical success rate without the major complication. In addition, the portal vein thrombosis resolved during the follow-up and three (27\%) patients later received the liver transplant without any early complications such as portal vein stenosis. ${ }^{8}$

The primary complication associated with the transsplenic approach is hemorrhage from the splenic puncture site and tract. ${ }^{4}$ In study involving 61 patients who received PVR-TIPS due to portal vein thrombosis, perisplenic hemorrhagic occurred in 2 patients who required transfusion of packed red blood cells. Neither patient required arterial embolization or splenectomy due to bleeding. ${ }^{7}$ Although embolization of the tract using the gelatin sponge is an established technique, gelatin sponge is not a permanent embolic material. The previous study, in which the puncture site and tract were embolized using permanent embolic materials such as coils and glue, reported no immediate or delayed bleeding from the spleen in all nine patients. ${ }^{4}$ Another potential complication associated with TIPS in chronic portal vein thrombosis is portal vein rupture during the angioplasty due to chronically scarred and fibrotic portal vein. If it were to occur, deployment of appropriately sized stent grafts can treat the rupture. ${ }^{7}$

In this patient, TIPS could have been tried using USG guidance, considering the fact that peripheral right portal vein was patent. Transhepatic USG guidance is less invasive, 
but visualization of puncture needle might be difficult because the operator cannot perform manipulation of puncture needle and control of USG probe simultaneously. Ileocolic vein approach using laparotomy may be an alternative access. However, surgical treatment is more invasive compared to the interventional approach.

Our patient received the TIPS procedure via trans-splenic access after failure to achieve intrahepatic parenchymal access to the portal vein via transjugular approach. TIPS access was finally achieved using the snare positioned within the thrombosed portal vein as the fluoroscopic target. After successful deployment of TIPS, no immediate or delayed bleeding from the splenic puncture site was observed. As demonstrated in our case, trans-splenic approach is a safe approach in the management of portal hypertension and its complications.

\section{References}

1 Fidelman N, Kwan SW, LaBerge JM, Gordon RL, Ring EJ, Kerlan RK Jr. The transjugular intrahepatic portosystemic shunt: an update. AJR Am J Roentgenol 2012;199(04):746-755

2 Zheng M, Chen Y, Bai J, et al. Transjugular intrahepatic portosystemic shunt versus endoscopic therapy in the secondary prophy- laxis of variceal rebleeding in cirrhotic patients: meta-analysis update. J Clin Gastroenterol 2008;42(05):507-516

3 Narahara Y, Kanazawa H, Fukuda T, et al. Transjugular intrahepatic portosystemic shunt versus paracentesis plus albumin in patients with refractory ascites who have good hepatic and renal function: a prospective randomized trial. J Gastroenterol 2011; 46(01):78-85

4 Chu HH, Kim HC, Jae HJ, et al. Percutaneous transsplenic access to the portal vein for management of vascular complication in patients with chronic liver disease. Cardiovasc Intervent Radiol 2012;35(06):1388-1395

5 Probst P, Rysavy JA, Amplatz K. Improved safety of splenoportography by plugging of the needle tract. AJR Am J Roentgenol 1978; 131(03):445-449

6 Zhu K, Meng X, Zhou B, et al. Percutaneous transsplenic portal vein catheterization: technical procedures, safety, and clinical applications. J Vasc Interv Radiol 2013;24(04):518-527

7 Thornburg B, Desai K, Hickey R, et al. Portal vein recanalization and transjugular intrahepatic portosystemic shunt creation for chronic portal vein thrombosis: technical considerations. Tech Vasc Interv Radiol 2016;19(01):52-60

8 Habib A, Desai K, Hickey R, et al. Portal vein recanalizationtransjugular intrahepatic portosystemic shunt using the transsplenic approach to achieve transplant candidacy in patients with chronic portal vein thrombosis. J Vasc Interv Radiol 2015;26(04): 499-506 